\title{
Herpes simplex virus latency-associated transcript gene function
}

\author{
Jennifer R Kent, Wen Kang, Cathie G Miller, and Nigel W Fraser \\ Department of Microbiology, University of Pennsylvania School of Medicine, Philadelphia, Pennsylvania, USA
}

\begin{abstract}
A major area of interest in the study of herpes simplex virus type 1 (HSV-1) involves the persistence of the virus within a latent state in neuronal cells of infected humans. The latency-associated transcripts (LATs) are believed to play a key role during HSV-1 latency. This review will discuss the most recent findings on the involvement of the LAT region with apoptotic pathways and how this relates to other potential functions of the LATs. Journal of NeuroVirology (2003) 9, 285-290.
\end{abstract}

Keywords: animal models; apoptosis; HSV; latency

\section{Introduction}

The LATs were first described by in situ hybridization in 1984 (Stroop et al, 1984), and 3 years later, they were also detected by Northern blotting (Spivack and Fraser, 1987; Stevens et al, 1987). The LAT region encodes for multiple transcripts, including the $8.3-\mathrm{kb}$ primary transcript and two stable introns of $2.0 \mathrm{~kb}$ and $1.5 \mathrm{~kb}$ (Farrell et al, 1991; Zwaagstra et al, 1990). The 2.0 kb LAT intron has been widely studied because it is the only abundant transcript found during the latent phase of an HSV-1 infection. HSV-1 infection involves both latent and lytic phases of infection. During a HSV-1 lytic infection, viral gene expression occurs in a cascade that is initiated by immediateearly gene transcription, followed by early and then late gene transcription, as first defined in cell culture (Honess and Roizman, 1974). The LAT gene, as well as being expressed during latency, is expressed late in the infectious cycle (Spivack and Fraser, 1988). Following lytic infection, HSV-1 infects neuronal cells associated with the sites of primary infection and there establishes a latent infection, which can then reactivate when the infected cells are exposed to various forms of stress (for review, see Roizman and Sears, 1995). The fact that all HSV isolates, whether HSV-1 or HSV-2, encode a LAT transcript containing

Address correspondence to Nigel W. Fraser, Department of Microbiology, University of Pennsylvania School of Medicine, 319 Johnson Pavilion, 3610 Hamilton Walk, Philadelphia, PA 191046076, USA. E-mail: nfraser@mail.med.upenn.edu

Received 18 November 2002; accepted 27 November 2002. a stable intron suggests that the LATs must have some importance to the virus. Deletion mutagenesis is frequently used in experiments to determine the role of the LATs during infection. The fact that many deletion mutants have been made in the LAT gene region and little indication of a function for the LAT gene detected merely highlights the strengths and weakness of deletion mutagenesis-it is very powerful in locating genetic elements, but it is dependant on the knowledge of a phenotype for the genetic element.

\section{Neuronal survival}

Programmed cell death, or apoptosis, is a controlled event that is involved in many processes, such as tissue development, immune system management, and host defense. Apoptosis is characterized by several morphological changes, such as chromatin condensation, DNA fragmentation, membrane blebbing, cell shrinkage, and formation of apoptotic bodies (for review, see Kerr et al, 1972). Apoptosis can rid the body of cells that carry damaged DNA or are infected by viruses. Thus, many viruses have evolved the ability to block apoptosis during viral infection of host cells, including adenovirus and several herpes viruses (for review, see Hay and Kannourakis, 2002). Recent literature has demonstrated that HSV-1 has multiple antiapoptotic genes, including ICP27, ICP22, US3, US5, ICP4, and most recently the region encoding the LATs (Aubert and Blaho, 2001).

Many viral genes appear to contribute to the ability of HSV-1 to block apoptosis in lytically infected cells. 
The LAT expression during latency suggests that their function would be important during latency, and possibly early reactivation. The ability to block induction of apoptosis would be extremely advantageous to the virus during latency, specifically to aid the virus in perpetuating its latent state.
An antiapoptotic function of the LATs was first suspected after observations of increased neurovirulence of a LAT mutant virus in rabbits (Perng et al, 1999). In the next few years, several papers were published using LAT deletion mutants to characterize the involvement of the LATs with apoptosis in vivo (Figure 1).
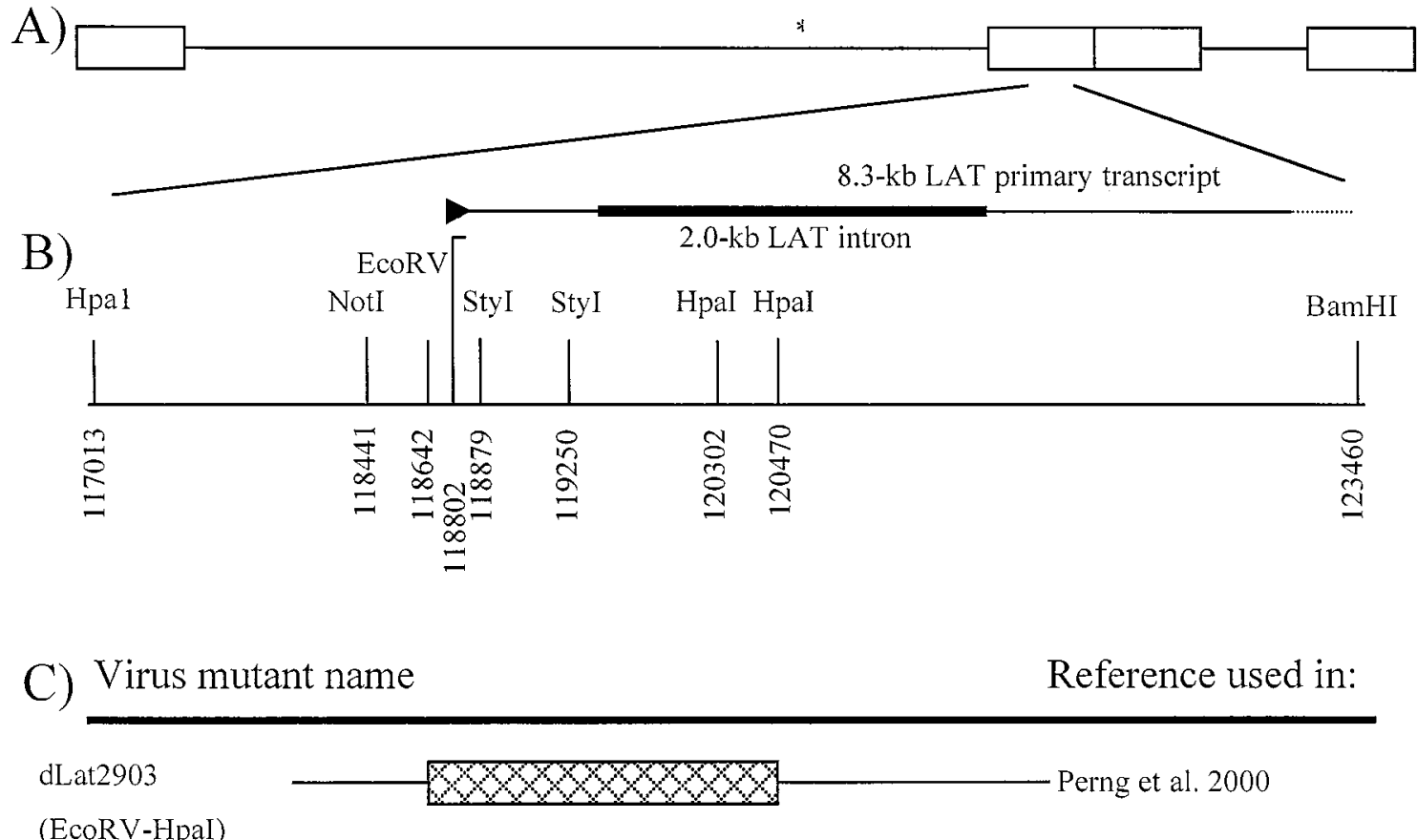

(EcoRV-HpaI)

$17 \mathrm{AH}$ Thompson and Sawtell 2001

(117674-119638)

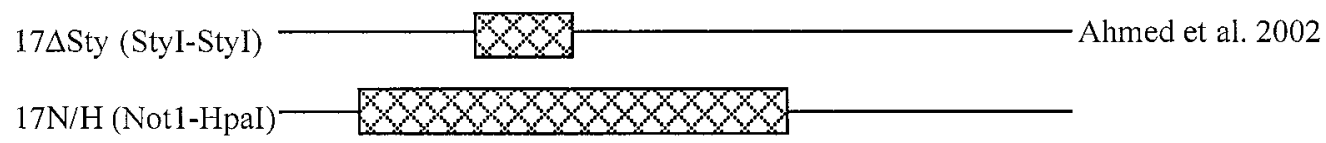

Rescued virus name

dLat3.3A(117011-120302)

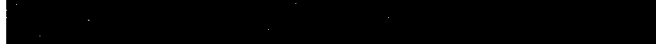
Inman et al. 2001

dLat2.6A(117011-119613)

dLat2.5A(117011-119463)

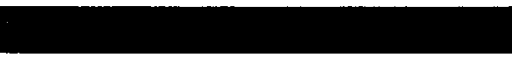

dLat1.8A(117011-118878)

Figure 1 Map of HSV-1 virus constructs involving genetic alterations within the LAT region. (A) Diagram of the genomic structure of HSV-1. (B) Expanded view of the LAT region of the genome. (C) Virus mutants containing deleted sequences are denoted with hatched boxes. Solid boxes indicate LAT sequence that was replaced ectopically between the UL37 and UL38 genes (approximate location marked with an asterisk) found within the unique long region of the dLAT2903 mutant virus genome. 
Perng et al (2000a) found significant numbers of apoptotic neurons in rabbit trigeminal ganglia (TGs) 7 days post infection when infected with dLAT2903, a virus with a deletion of $1828 \mathrm{bp}$ from within the LAT region. This deletion includes the LAT promoter and the $5^{\prime}$ coding region of the 2.0-kb stable LAT intron, resulting in loss of the LAT RNAs (Perng et al, 2000a). Apoptotic neurons were identified by terminal deoxynucleotidyl transferase-mediated deoxyuridine triphosphate nick-end labeling (TUNEL) staining and poly(ADP-ribose) polymerase (PARP) cleavage, two well-characterized techniques for detecting the occurrence of apoptotic cells. In acutely infected mouse TG sections at 3 and 6 days following infection, Ahmed et al (2002) saw no difference in apoptosis between a wild-type $17+$ virus and a LAT exon 1 deletion virus $(17+\Delta$ Sty) as measured by the DeadEnd colorimetric assay (Promega). However, tissues infected with a larger deletion mutant $(17+$ NotI-HpaI), which eliminates the LAT promoter, exon 1 , and the $5^{\prime}$ end of the 2.0-kb LAT, demonstrated increased apoptotic staining associated with HSV-1-infected cells (Ahmed et al, 2002).

The notion that the LATs have an antiapoptotic function has been challenged by Thompson and Sawtell (2000, 2001). Using the 17-AH virus, which is a LAT deletion mutant lacking the entire LAT promoter and $827 \mathrm{bp}$ of the $5^{\prime}$ end of the primary LAT transcript, they found increased neuronal death in mouse TGs infected with the LAT mutant virus compared to those infected with the parental strain using the contextual analysis of DNA (CXA-D) technique (Thompson and Sawtell, 2001). However, in contrast to the extensive apoptosis observed by Perng et al in rabbit TGs infected with a LAT mutant, Sawtell and Thompson found very few cells in the mouse TGs undergoing apoptosis during the acute stage of infection as measured by TUNEL staining. In addition, the cells that were apoptotic appeared to be non-neuronal, and possibly were infiltrating immune cells. The discrepancy in the apoptosis reported by these two groups may be partially due to the difference in the animal models employed. Controversy also exists regarding the ability of the tests used to detect apoptosis within the rabbit model that was used by Perng et al (2000a) and Thompson and Sawtell (2000).

Currently, it cannot be ruled out that the LAT promotes neuronal survival through mechanisms other than antiapoptosis. Using CXA-D, Sawtell (1997) and Thompson and Sawtell (1997) showed LAT mutants established latency in fewer numbers of cells isolated from mice infected at least 30 days prior. They hypothesized that the LATs promote neuronal survival by preventing high multiplicity of infections, possibly by down-regulating viral gene expression during productive infections. This hypothesis concerning LAT down-regulation of lytic genes has also been proposed by others (Garber et al, 1997).

To further characterize the involvement of the LATs in apoptosis, fragments of the LAT region were tested for their ability to block apoptosis caused by exogenous agents in various cell lines (Figure 1). Initial studies used an expression plasmid (APALAT) that contained $2358 \mathrm{bp}$ of the LAT region, including the region deleted within the dLAT2903 LAT mutant virus (Perng et al, 2000a). After transfection with the APALAT plasmid, apoptosis was induced in IMR-90 and CV-1 cells using $\mathrm{C}_{6}$-ceramide or $\mathrm{FB}_{1}$ and neuro$2 \mathrm{~A}$ cells with etoposide. The region of LAT expressed by this plasmid was able to protect cells from apoptosis induced by any of the agents (Perng et al, 2000a). Further experiments followed, including an in depth analysis of the LAT regions important for protection from a variety of apoptosis-inducing agents. Plasmids containing at least the first $811 \mathrm{bp}$ of the LAT primary transcript along with the promoter are sufficient for protection from apoptosis as induced by chemicals or expression of Bax, a proapoptotic protein (Inman et al, 2001). In another study, expression of different regions of the $5^{\prime}$ LAT demonstrated that the exon 1 region is more likely to be involved in protecting cells than the stable 2.0-kb LAT intron when apoptosis was induced by caspase 8 expression (Ahmed et al, 2002).

The data discussed above support two models of neuronal survival. One model suggests that the LATs possess antiapoptotic activity that protects HSV-infected neurons from detection and deletion by apoptotic signaling pathways. The other model suggests that the LATs affect the process perhaps by down-regulating viral gene expression during establishment of latency, leading to large numbers of latently infected neurons. When the LATs are not present, infected neurons will continue with a lytic program of viral transcription, which results in the destruction of neuronal cells.

\section{Role of LATs in latency and reactivation}

Several lines of evidence indicated that the LATs may function to down-regulate productive infections and suppress viral replication. In situ hybridization studies of acutely infected mouse TGs demonstrated several fold increases in the number of cells expressing viral early and immediate-early genes with a LAT mutant compared to those infected with the wildtype (Garber et al, 1997). It has been suggested that increases in the numbers of cells expressing lytic genes could result in a greater immune response by the host, leading to a decrease in the numbers of latent cells (Chen et al, 2000). Furthermore, Mador et al (1998) showed that viral replication was inhibited in cell lines expressing the LATs, and the LATs appeared to have a suppressive effect on the expression of viral immediate early genes ICP0, ICP4, and ICP27.

Upon reaching sensory ganglia, the virus within infected neurons either continues along the lytic life cycle, leading to a productive infection, or enters a latent state, during which most of the viral genes are 
silenced. The mechanism by which the virus enters and maintains its latent state is unclear. There is evidence that the choice of forming lytic or latent infection occurs soon after entry of the neuronal cell nucleus (Steiner et al, 1990). It has been proposed that interaction between the virus and the neuronal fast axonal transport mechanisms may result in inefficient transfer of tegument proteins to the nucleus, which results in inefficient up-regulation of viral gene expression, leading to establishment of a latent infection (Roizman and Sears, 1987). It is thought that the LATs play a role in promoting the efficient establishment of latency (Sawtell and Thompson, 1992; Thompson and Sawtell, 1997). Although a number of earlier studies showed that mutations in the LAT region had no effect on the level of latent viral DNA (Sederati et al, 1989), several recent reports reveal that LAT mutant viruses establish latency in fewer number of cells in both mouse (Thompson and Sawtell, 1997; Sawtell and Thompson, 1992) and rabbit (Perng et al, 2000b) latency models. A small deletion in the LAT promoter alone can cause a reduction in the number of latently infected cells, indicating that the transcription of the LATs is crucial for establishing latency (Sawtell and Thompson, 1992).

The maintenance of viral latency is not well understood. Because the 2.0-kb LAT intron is abundantly present during latency, and the $3^{\prime}$ part of the $2.0-\mathrm{kb}$ LAT intron overlaps the viral immediate early gene ICP0, it has been hypothesized that the LATs play a role in maintaining viral latency by acting as antisense RNAs to the immediate early gene ICP0 to block viral replication (Stevens et al, 1987). In addition, one study showed that the LAT intron inhibits the transactivating activity of ICP0 in a transient transfection assay (Farrell et al, 1991). It has been long thought that during latency, there is no transcription of the viral genome except for from the LAT region. However, recent studies by several groups have revealed low but detectable levels of lytic viral gene mRNA during latency (Chen et al, 1997; Kramer and Coen, 1995). Interestingly, this study by Chen et al showed an increase of several folds in the transcripts of viral immediate early gene ICP4 and early gene TK during latent infection with a LAT mutant virus (Chen et al, 1997). However, in other studies, the level of the ICP0 transcript was not changed with the LAT mutant, arguing against the theory that the LATs act antisense to ICP0 (Chen et al, 2002). Moreover, the increase of the ICP4 transcript was accompanied by a decrease in anti-ICP4, a viral transcript most likely originating from the LAT promoter (Chen et al, 1997). Therefore, the LAT transcripts may have a role in down-regulating the expression of ICP4, rather than affecting ICP0. By examining a large number of infected mouse TG sections, Feldman et al (2002) found a small number of cells expressing productive cycle genes during latency. The significance of this observation is unclear. These cells expressing lytic genes may represent cells where the virus reactivates with low levels of gene expression, but reactivation does not gain momentum leading to a recrudescence (as measured by titer of infectious virus) (Feldman et al, 2002).

In animal models, reactivation of HSV-1 can occur in response to any one of several stressful stimuli (mouse and rabbit models) or may be spontaneous (rabbit model). One group has suggested the presence of a LAT protein encoded from an open reading frame (ORF) within the 2.0-kb intron region of the LAT gene. They believe that this tightly regulated, and thus difficult to detect, protein acts somewhat similarly to ICP0, specifically involving lytic gene enhancement and interaction with cellular factors involved in promoting transcription (Thomas et al, 1999, 2002). Expression of this potential LAT protein during the early stages of reactivation could be important for initiating lytic gene expression from latent viral genomes. Previous work indicated that the first $1.5 \mathrm{~kb}$ of the LAT region is necessary for spontaneous reactivation in rabbits (Perng et al, 1996), but the first 811 bp can partially restore this phenotype to an HSV-1 LAT-null mutant (Drolet et al, 1999). Interestingly, these LAT sequences correspond to regions used in expression plasmids to demonstrate the LAT's ability to convey protection from apoptoticinducing agents (Inman et al, 2001). The latencyrelated gene of bovine herpes virus 1, which also has been shown to possess antiapoptotic characteristics, can recover the spontaneous reactivation phenotype of a LAT-null mutant (Perng et al, 2002). These associations lead to speculation of a correlation between the LATs' antiapoptotic ability and the role of the LATs in reactivation of the virus, although the apoptotic

Table 1 Proposed LAT functions

\begin{tabular}{lll}
\hline Role of LAT & \multicolumn{1}{c}{ Mechanism } & \multicolumn{1}{c}{ Evidence } \\
\hline Neuronal survival & Antiapoptotic & Perng et al, 2000; Ahmed et al, 2002 \\
& Modulate acute infection & Garber et al, 1997; Mador et al, 1998 \\
Maintenance of latency & Immune modulation & Chen et al, 2000 \\
& Antisense to ICP0 & Farrell et al, 1991; Stevens et al, 1987 \\
Reactivation & Antisense to ICP4 & Chen et al, 1997 \\
& LAT-encoded protein promotes & Thomas et al, 1999; Thomas et al, 2002 \\
& Aytic gene transcription & Inman et al, 2001 \\
\hline
\end{tabular}


mapping data would suggest that the LAT exon rather than the intron is the functional molecule.

In summary, at present there are two main hypotheses regarding the role of the LATs in neuronal survival: (1) the LATs promote cell survival by preventing infected neurons from undergoing apoptosis or (2) the LATs may down-regulate viral gene expres-

\section{References}

Ahmed M, Lock M, Miller CG, Fraser NW (2002). Regions of the herpes simplex virus type 1 latency-associated transcript that protect cells from apoptosis in vitro and protect neuronal cells in vivo. J Virol 76: 717-729.

Aubert M, Blaho JA (2001). Modulation of apoptosis during herpes simplex virus infection in human cells. Microbes Infect 3: 859-866.

Chen SH, Garber DA, Schaffer PA, Knipe DM, Coen DM (2000). Persistent elevated expression of cytokine transcripts in ganglia latently infected with herpes simplex virus in the absence of ganglionic replication or reactivation. Virology 278: 207-216.

Chen S-H, Kramer MF, Schaffer PA, Coen DM (1997). A viral function represses accumulation of transcripts from productive-cycle genes in mouse ganglia latently infected with herpes simplex virus. J Virol 71: 5878-5884.

Chen SH, Lee LY, Garber DA, Schaffer PA, Knipe DM, Coen DM (2002). Neither LAT nor open reading frame P mutations increase expression of spliced or intron-containing ICP0 transcripts in mouse ganglia latently infected with herpes simplex virus. J Virol 76: 4764-4772.

Drolet BS, Perng GC, Villosis RJ, Slanina SM, Nesburn AB, Wechsler SL (1999). Expression of the first $811 \mathrm{nu}$ cleotides of the herpes simplex virus type 1 latencyassociated transcript (LAT) partially restores wild-type spontaneous reactivation to a LAT-null mutant. Virology 253: 96-106.

Farrell MJ, Dobson AT, Feldman LT (1991). Herpes simplex virus latency-associated transcript is a stable intron. Proc Natl Acad Sci USA 88: 790-794.

Feldman LT, Ellison AR, Voytek CC, Yang L, Krause P, Margolis TP (2002). Spontaneous molecular reactivation of herpes simplex virus type 1 latency in mice. Proc Natl Acad Sci USA 99: 978-983.

Garber DA, Schaffer PA, Knipe DM (1997). A LAT associated function reduces productive-cycle gene expression during acute infection of murine sensory neurons with herpes simplex virus type 1. J Virol 71: 5885-5893.

Hay S, Kannourakis G (2002). A time to kill: viral manipulation of the cell death program. J Gen Virol 83: 1547-1564.

Honess RW, Roizman B (1974). Regulation of herpes macromolecular synthesis. I. Cascade regulation of the synthesis of three groups of viral proteins. J Virol 14: 8-19.

Inman M, Perng GC, Henderson G, Ghiasi H, Nesburn AB, Wechsler SL, Jones C (2001). Region of herpes simplex virus type 1 latency-associated transcript sufficient for wild-type spontaneous reactivation promotes cell survival in tissue culture. J Virol 75: 3636-3646.

Kerr JF, Wyllie AH, Currie AR (1972). Apoptosis: a basic biological phenomenon with wide-ranging implications in tissue kinetics. Br J Cancer 26: 239-257.

Kramer MF, Coen DM (1995). Quantification of transcripts from the ICP4 and thymidine kinase genes in mouse sion, leading to a milder infection and less neuronal damage. Neither hypothesis is mutually exclusive, and experimental support for both hypotheses has been shown (see Table 1). However, the mechanism by which the LAT gene functions to prevent cell death and decrease viral gene expression remains ill defined. ganglia latently infected with herpes simplex virus. J Virol 69: 1389-1399.

Mador N, Goldenberg D, Cohen O, Panet A, Steiner I (1998). Herpes simplex virus type 1 latency-associated transcripts suppress viral replication and reduce immediateearly gene mRNA levels in a neuronal cell line. J Virol 72: 5067-5075.

Perng GC, Ghiasi H, Slanina SM, Nesburn AB, Wechsler SL (1996). The spontaneous reactivation function of the herpes simplex virus type 1 LAT gene resides completely within the first 1.5 kilobases of the 8.3-kilobase primary transcript. J Virol 70: 976-984.

Perng G-C, Jones J, Ciacci-Zanella J, Stone M, Henderson G, Yukht A, Slanina SM, Hofman FM, Ghiasi H, Nesburn AB, Wechsler SL (2000a). Virus-induced neuronal apoptosis blocked by the herpes simplex virus latency associated transcript. Science 287: 15001503.

Perng GC, Maguen B, Jin L, Mott KR, Osorio N, Slanina SM, Yukht A, Ghiasi H, Nesburn AB, Inman $M$, Henderson G, Jones C, Wechsler SL (2002). A gene capable of blocking apoptosis can substitute for the herpes simplex virus type 1 latency-associated transcript gene and restore wild-type reactivation levels. J Virol 76: $1224-1235$.

Perng GC, Slanina SM, Yukht A, Drolet BS, Keleher W, Jr., Ghiasi H, Nesburn AB, Wechsler SL (1999). A herpes simplex virus type 1 latency-associated transcript mutant with increased virulence and reduced spontaneous reactivation. J Virol 73: 920-929.

Perng GC, Slanina SM, Yukht A, Ghiasi H, Nesburn AB, Wechsler SL (2000b). The latency-associated transcript gene enhances establishment of herpes simplex virus type 1 latency in rabbits. J Virol 74: 1885-1891.

Roizman B, Sears AE (1987). An inquiry into the mechanisms of herpes simplex virus latency. Ann Rev Microbiol 41: 543-571.

Roizman B, Sears AE (1995). Herpes simplex viruses and their replication. In: Virology. Fields BN, Knipe DM, Howley PM (eds). New York: Raven Press, pp 22312296.

Sawtell NM (1997). Comprehensive quantification of herpes simplex virus latency at the single-cell level. $J$ Virol 71: 5423-5431.

Sawtell NM, Thompson RL (1992). Herpes simplex virus type 1 latency-associated transcription unit promotes anatomical site-dependent establishment and reactivation from latency. J Virol 66: 2157-2169.

Sederati F, Izumi KM, Wagner EK, Stevens JG (1989). Herpes simplex virus type 1 latency-associated transcription plays no role in establishment or maintenance of a latent infection in murine sensory neurons. J Virol 63: 4455-4458. 
Spivack JG, Fraser NW (1987). Detection of herpes simplex type 1 transcripts during latent infection in mice. $J$ Virol 61: 3841-3847.

Spivack JG, Fraser NW (1988). Expression of herpes simplex virus type 1 (HSV-1) latency-associated transcripts in the trigeminal Ganglia of mice during acute infection and reactivatioon of latent infection. J Virol 62: 14791485.

Steiner I, Spivack JG, Deshmane SL, Ace CI, Preston CM, Fraser NW (1990). A herpes simplex virus type $1 \mathrm{mu}-$ tant containing a non-transinducing Vmw65 protein establishes latent infection in vivo in the absence of viral replication and reactivates efficiently from explanted trigeminal ganglia. J Virol 64: 1630-1638.

Stevens JG, Wagner EK, Devi-Rao GB, Cook ML, Feldman LT (1987). RNA complementary to a herpes virus gene mRNA is prominent in latently infected neurons. Science 235: 1056-1059.

Stroop WG, Rock DL, Fraser NW (1984). Localization of herpes simplex virus in the trigeminal and olfactory systems of the mouse central nervous system during acute and latent infections by in situ hybridization. Lab Invest 51: $27-38$

Thomas SK, Gough G, Lachman DS, Coffin RS (1999). Herpes simplex virus latency-associated transcript encodes a protein which greatly enhances virus growth, can compensate for deficiencies in immediate early gene expression, and is likely to function during reactivation from virus latency. J Virol 73: 6618-6625.

Thomas SK, Lilley CE, Latchman DS, Coffin RS (2002). A protein encoded by the herpes simplex virus (HSV) type 12 -kilobase latency-associated transcript is phosphorylated, localized to the nucleus, and overcomes the repression of expression from exogenous promoters when inserted into the quiescent HSV genome. J Virol 76: 4056-4067.

Thompson RL, Sawtell NM (1997). The herpes simplex virus type-1 latency-associated transcript gene regulates the establishment of latency. J Virol 71: 5432-5440.

Thompson RL, Sawtell NM (2000). HSV latency-associated transcript and neuronal apoptosis. Science 289: 1651.

Thompson RL, Sawtell NM (2001). Herpes simplex virus type 1 latency-associated transcript gene promotes neuronal survival. J Virol 75: 6660-6675.

Zwaagstra J, Ghiasi H, Slanina SM, Nesburn AB, Wheatley SC, Lillycrop K, Wood J, Latchman DS, Patel K, Wechsler SL (1990). Activity of herpes simplex virus type 1 latency associated transcript (LAT) promoter in neuron-derived cells: evidence for neuron specificity and for a large LAT transcript. J Virol 64: 5019-5028. 